\title{
Channel Modelling of Underwater Communication Network with AUVS
}

\author{
R.S Deepak Ram, Vijayalakshmi.P, Venkataraman Padmaja, T. Jaya, V. Rajendran
}

\begin{abstract}
Underwater communication network always consists of static, slow, and fast-movingnodeslimitedto a few tens of nodes. In generally, network is governed by acoustic system as a communication model. This makes the system as BW limited, spectrum inefficiency etc. In addition, the sensors are autonomously put in the sea bottom is battery supported and so the energy optimization is also needed to be addressed in this paper. The paper explains on detailed comparison on performance of different channel modelling of. Ainslie and McColm Model, Ambient Noise Model, Thorp Model \&. Fisher \& Simmons Model. The analysis shows that Ainslie-McColm and Fisher \& Simmons gives better energy optimization.
\end{abstract}

Index Terms: Acoustic ocean modelling, underwater technology, wireless communication network.

\section{INTRODUCTION}

Oceanographic applications like harvesting mines in the deep sea (Deepak et al, 2001), disaster warning systems (Nayak et.al 2009), prediction of positional accuracy system for moving nodes (Kongsberg Simrad) etc. consists of a specialized wireless communication network that are limited to a few tens of nodes (static, slow, and fast-moving nodes). (Heidemann et al 2006, Sozer et al, 2000, Heidemann et al , 2012, Kong et al, 2005). In this application slow and fastmoving nodeare utilized byAUV, ROV, as a survey system for the operation of search and retrieve.(Ludvigsen et al.,2013)It is well known that the acoustic wave is used for the transmitting data because of electromagnetic radiation suffers high attenuation in sea water the acoustic systems and sensing systems is limited by its bandwidth, spectrum efficiency etc. (Jiang, Z.2008).

\section{RELATED WORK}

In addition, since the sensors putting in underwater is autonomous in sea bottom the sensors are mostly supported by battery pack energy supply. Hence the energy optimization in such a network becomes a prominent variant to be addressed as equal to spectrum efficiency, BW limitation etc.

Revised Manuscript Received on October 15, 2019.

R. S Deepak Ram, Research Scholar, Dept. of ECE, VISTAS, Pallavaram, Chennai, Tamilnadu, India.(Email: deepakramrs@gmail.com)

Vijayalakshmi. P, Asst. professor, Dept. of ECE, Vels Institute of Science, Technology \& Advanced Studies (VISTAS), Pallavaram, Chennai, Tamilnadu, India.(Email: viji.se@ velsuniv.ac.in)

Venkataraman Padmaja, Research Scholar, Sathyabama Institute of Science and Technology, Chennai, Tamilnadu, India.(Email: ktananya@gmail.com)

Dr. T. Jaya, Asst. professor, Dept. of ECE, Vels Institute of Science, Technology \& Advanced Studies (VISTAS), Pallavaram, Chennai, Tamilnadu, India.(Email: Jaya.se@ velsuniv.ac.in)

Dr. V. Rajendran, Professor and Director of Department of Electronics and Communication Engineering, VISTAS, Chennai, Tamil Nadu, India.(Email: drvrajen@gmail.com).
Before getting into the actual configuration, simulation of the smallest network having 7 to 10 nodes and its behavior for the above parameters for any oceanographic application becomes primary aspect of research. The special purpose tools like AquaTools, Aquasim etc. are used for carrying out simulation of these models incorporateunderwater parameters in addition to any terrestrial network simulator (Sehgal, et al, 2010).

Simulation model should majorly address the channel characterization by using appropriate channel model because the composite and compounded nature of the undersea water propagation. Since the medium is so complex not a single type of model can be a total success to give a final answer (Sehgal,et al . 2010).

Hence the study of channel modelling with reference to network performance for a configured, simulated underwater sensor networkis required to be carried out (Vijayalakshmi, et al, 2016 \& 2018).

Therefore, proposed in the paper are

a) Practical configuration for application.

b) Right utilization of tools

c) Different channel characterization model

d) Network performance like SNR, PDR, throughput.

e) Energy optimization.

Subsequent sections explain all five-different area with qualitative and quantitative results and concluded with a summary. This innovate approach probably can be proposed for any type and any number of nodes configuration later for further investigation.

\section{SYSTEM OVERVIEW}

For any oceanographic applications the minimum required network model to be considered (Das, et al 2017) has one surface sink, one surface station, 4 static nodes, two AUV's, 1 slow moving node and 1 fast moving node. With above basic configuration the total model and simulation are carried out.

Published By: Blue Eyes Intelligence Engineering 


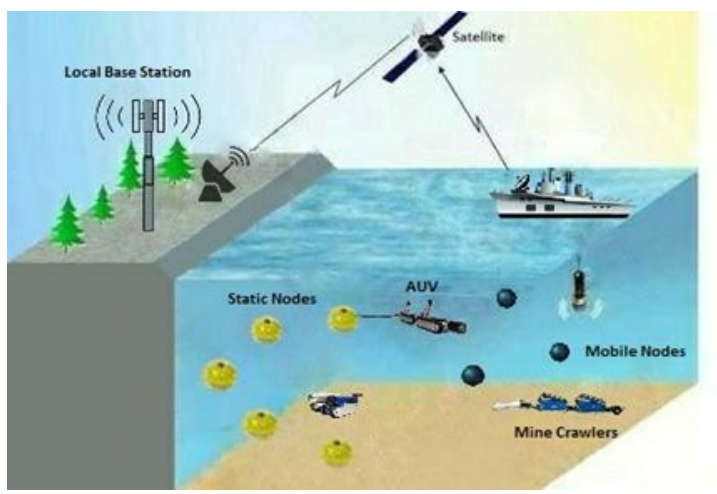

Fig. 1. Underwater Network Model

\section{A. TOOLS USED:}

As the area of underwater acoustics communication is new few efforts have been made towards the simulation and lab testing of underwater acoustic networks, tools like ns miracle, OPNETs, MATLAB, Aqua Tools, etc. (Sehgal, et al . 2010) are available. Each of the above tools has their own advantages and disadvantages. For our network design and simulation, Aquasim is found to be the best suited simulation tools. (Das, et al 2017).

Aquasim (Das, et al2017)isdeveloped on the existingNS-2 simulator. Using Aquasim we can simulate underwater sensor nodes, traffic patterns,acoustic channels, and networking protocols.Object-oriented style used is used for designing and supports 3D deployment. Independent parallel CMU wireless package in Aquasim shown in fig. 2. Due to this any changes done in Aqua-Sim does not hamper with otherCMU packages in NS-2.

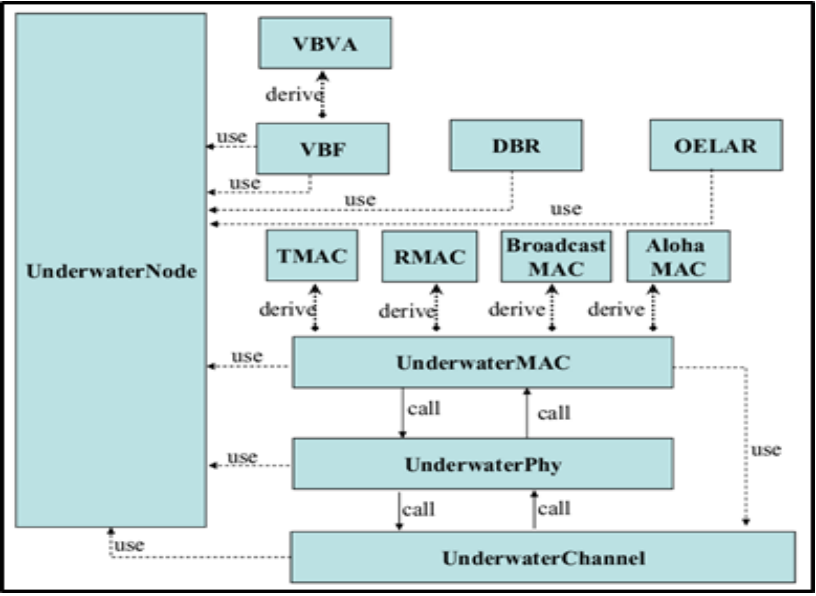

Fig. 2. Aqua-Sim architecture

Fig.2 shows the system architecture of Aquasim and selfexplanatory protocol stack implemented in Aquasim, which users can use to test different protocols at each layer such as reliable data transfer, routing, and MAC layer protocols for the configuration considered above. (Vijayalakshmi, et al, $2016 \& 2018)$.

\section{B. CHANNEL CHARACTERIZATION}

Performance of Underwater acoustic channel depends on the propagation model. Propagation model changes due to the underwater medium (location also) characteristics chosen. Differentattenuation models are discussed on which different propagation models are based.

The models that have considered for the study:

1. Thorp Model
2. Fisher\& Simmons Model

3. Ainslie and McColm Model

4. Ambient Noise Model.

a. Thorp Model

This is a simple model, only the frequency is utilized, and relaxation frequencies effects, acidity and salinity of the ocean are ignored. So the medium of study is simple.

$$
\alpha=\frac{0.1 f^{2}}{1+f^{2}}+\frac{40 f^{2}}{4100+f^{2}}+2.75 \times 10^{-4} \cdot f^{2}+0.003
$$

\section{b. Fisher \&Simmons Model}

In this model the temperature and depth effects are considered along with relaxation frequencies effects caused by magnesium sulphate and boric acid (Ma, et al. 2013).

$$
\alpha=A_{1} P_{1} \frac{f_{1} f^{2}}{f_{1}^{2}+f^{2}}+A_{2} P_{2} \frac{f_{2} f^{2}}{f_{2}^{2}+f^{2}}+A_{3} P_{3} f^{2}
$$

Here

$\mathrm{A} 1, \mathrm{~A} 2$ and $\mathrm{A} 3$ are temperature functions

P1, P2 and P3 are constant equilibrium pressurefunctions.(Sehgal, et al. 2009).

These are represented as:

$$
\begin{aligned}
& A_{1}=1.03 \times 10^{-8}+2.36 \times 10^{-10} \cdot T-5.22 \times 10^{-12} \cdot T^{2} \\
& A_{2}=5.62 \times 10^{-8}+7.52 \times 10^{-10} \cdot T \\
& A_{3}=\left[55.9-2.37 \cdot T+4.77 \times 10^{-2} \cdot T^{2}-3.48 \times 10^{-4} \cdot T^{3}\right] \cdot 10^{-15} \\
& f_{1}=1.32 \times 10^{3}(T+273.1) e^{\frac{-1500}{T+27.1 .3}} \\
& f_{2}=1.55 \times 10^{7}(T+273.1) e^{\frac{-453}{T+272.1}} \\
& P_{1}=1 \\
& P_{2}=1-10.3 \times 10^{-4} \cdot P+3.7 \times 10^{-7} \cdot P^{2} \\
& P_{3}=1-3.84 \times 10^{-4} \cdot P+7.57 \times 10^{-8} \cdot P^{2}
\end{aligned}
$$

This model operates at depth up to $8 \mathrm{~km}$ and salinity restriction of $35 \mathrm{ppm}$, and $\mathrm{PH}$ should be set at 8 .

\section{c. Ainslie-McColm Model:}

This model proposed extra relaxations and simplifications as follows.

$$
\begin{aligned}
\alpha= & 0.106 \frac{f_{1} f^{2}}{f_{1}^{2}+f^{2}} e^{\frac{p H-8}{0.56}} \\
& +0.52\left(1+\frac{T}{43}\right)\left(\frac{S}{35}\right) \frac{f_{2} f^{2}}{f_{2}^{2}+f^{2}} e^{\frac{-D}{6}} \\
& +4.9 \times 10^{-4} f^{2} e^{-\left(\frac{T}{29}+\frac{D}{17}\right)}
\end{aligned}
$$

In the Ainslie- McColm model effects of acidity of sea water are considered and it is a function of depth unlike the previous model which is a function of pressure.

Equation for $\mathrm{f} 1$ and $\mathrm{f} 2$ are as follows:

$$
\begin{aligned}
& f_{1}=0.78 \sqrt{\frac{S}{35}} e^{\frac{T}{25}} \\
& f_{2}=42 e^{\frac{T}{17}}
\end{aligned}
$$

\section{d. Ambient Noise Model}

The ambient noise is gaussian and has a continued power spectrum density. Turbulence, wind driven waves, shipping, and thermal noise are the most commonly considered noises. 


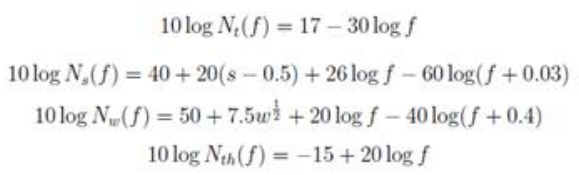

The ambient noise is colored, and at specific Heddeghem,2009)

The overall Noise p.s.d may be obtained from:

$$
N(f)=N_{t}(f)+N_{s}(f)+N_{w}(f)+N_{t h}(f)
$$

The noise p.s.d values along with the attenuation can help to characterize the channel performance (Rattaro et al. 2017). Using the above studied attenuation models, we predict the network performance. (Sehgal, et al. 2010, May).

\section{NETWORK PERFORMANCE \& RESULTS}

The network performance is evaluated for the parameters like SNR, packet delivery ratio, energy consumption, and throughput (Rajamohamed, 2012). The parameter Throughput has been processed with the number of correctly delivered packets per unit time. Fig 4 shows that Ainslie \& McColm model has the best throughput and the thorp model has the least throughput. After $1000 \mathrm{sec}$ of simulation thethroughput differencebetween thethree models is close to 300kbps. Sozer, E, et.al (2000).

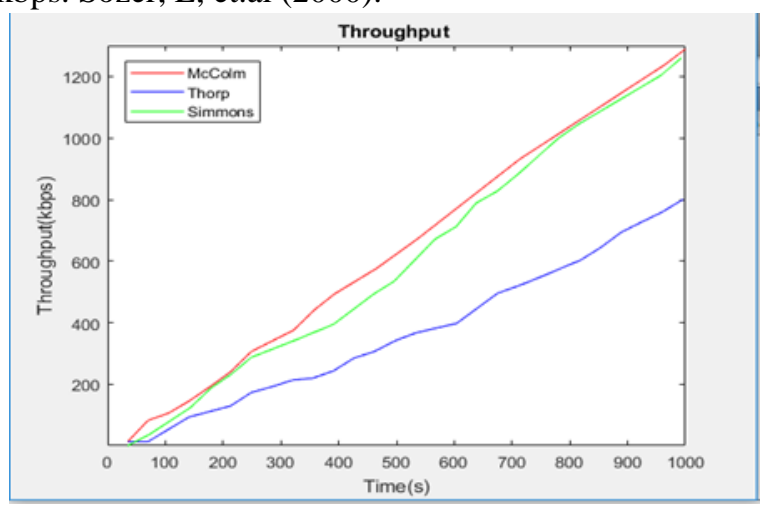

Fig. 3. Comparison graph for Throughput

The parameter calculated as a rate of receivedpackets at destination to the packets generated at the source is called packet delivery.From fig.5 Ainslie\& McColm model has the best packet delivery ratio. After $1000 \mathrm{sec}$ of simulation the difference in packet delivery ratio of the three models is close to 10

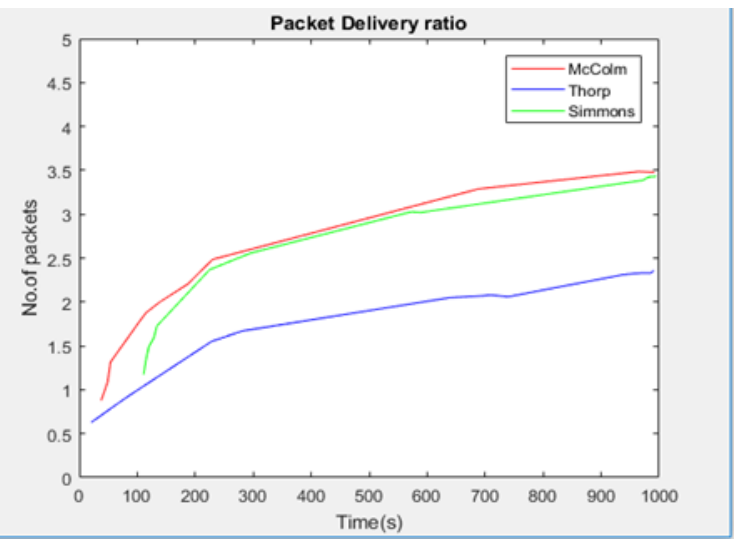

Fig. 4. Comparison graph for Packet delivery ratio frequencieshave pronounced effects different factors.(Van

The energy consumption is calculated as the total energy consumed by the whole network for transmission. From fig 6 Fisher \& Simmons model provides best energy consumption. After $1000 \mathrm{sec}$ of simulation the difference in energy consumption between the four models is close to 400joules.

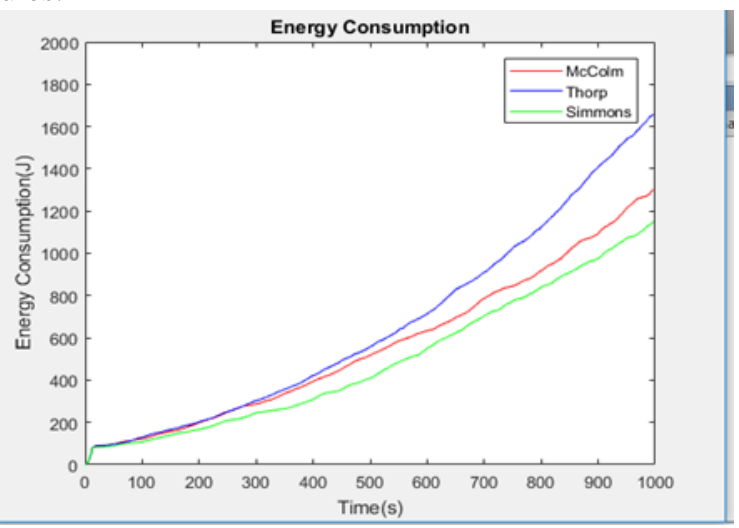

Fig. 5. Comparison graph for Energy consumption

The parameter SNR has been calculated as the ratio of number of actual packets transmitted to that of the noise packets that are transmitted. From fig $6 \mathrm{McColm}$ model has the least SNR of the three models. The difference in SNR between the Thorp model and Ainslie-McColm models is $9 \mathrm{~dB}$.

As discussed in the earlier section wireless sensor networks having nodes as mentioned in configuration are powered with limited energy irreplaceable batteries. To be able to have reliable and good communication as well as good network life the design of energy efficient network, should be implemented. For the above designed network, the Ainslie-McColm and Fisher-Simmons propagation model gives the best energy optimization. (Jones, et al 2001, Sehgal, et al. 2010).

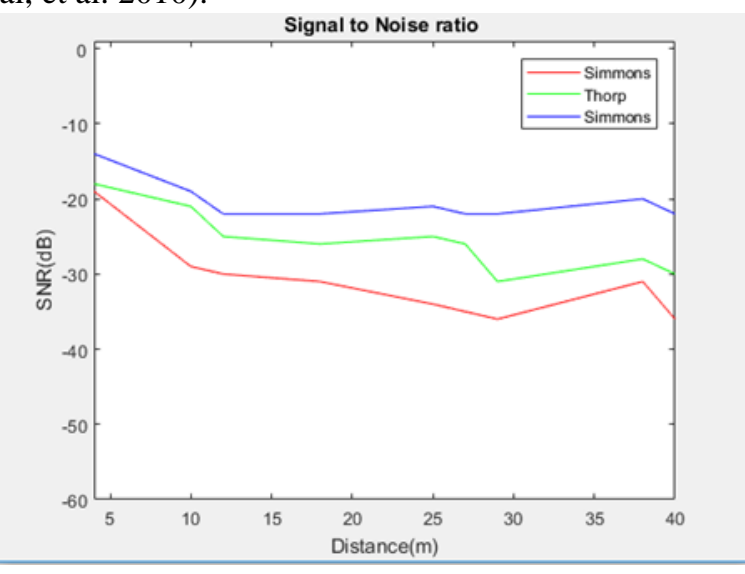

Fig. 6. Comparison graph for Signal to Noise ratio

\section{CONCLUSION}

It is clearly explained for the above configuration network performance improvement, in our opinion that the characteristic parameter with the appropriate model can yield quite good performance and however it can be noted that it is location specific in characterization. The further

Published By: 
growth can be emphasized for larger networks and validate through embedded emulator networks.

\section{REFERENCES}

1. Das, A. P., \& Thampi, S. M. (2017). Simulation tools for underwater sensor networks: a survey. Network Protocols and Algorithms, 8(4), 41-55

2. Deepak, C. R., Shajahan, M. A., Atmanand, M. A., Annamalai, K., Jeyamani, R., Ravindran, M., \& Schwarz, W. (2001, January), Developmental tests on the underwater mining system using flexible riser concept. In Fourth ISOPE Ocean Mining Symposium, International Society of Offshore and Polar Engineers.

3. Heidemann, J., Ye, W., Wills, J., Syed, A., \& Li, Y. (2006, April), Research challenges and applications for underwater sensor networking. In Wireless Communications and Networking Conference, 2006. WCNC 2006. IEEE (Vol. 1, pp. 228-235).

4. Heidemann, J., Stojanovic, M., \& Zorzi, M. (2012), Underwater sensor networks: applications, advances and challenges. Phil. Trans. R. Soc. A, 370(1958), 158-175.

5. Jiang, Z. (2008). Underwater acoustic networks-issues and solutions. International journal of intelligent control and systems, 13(3), 152-161.

6. Jones C.E, et al.;" A survey of energy efficient network protocol(I)", Wireless Networks, 7 (2001), pp 343-358

7. Kong, J., Cui, J. H., Wu, D., \& Gerla, M. (2005, October), Building underwater ad-hoc networks and sensor networks for large scale real-time aquatic applications. In Military Communications Conference, 2005. MILCOM 2005, IEEE (pp. 1535-1541).

8. Ludvigsen, M., Johnsen, G., Lagstad, P. A., Sorensen, A. J., \& Odegard, Ø. (2013, June), Scientific operations combining

9. ROV and AUV in the Trondheim Fjord. In OCEANS Bergen, 2013 MTS/IEEE (pp. 1-7).

10. Ma, W., Chai, F., Xiu, P., Xue, H., \& Tian, J. (2013). Modeling the long-term variability of phytoplankton functional groups and primary productivity in the South China Sea, "Journal of oceanography", 69 (5), 527-544.

11. Nayak, S., \& Kumar, S. (2009), Handbook on "Tsunami Early Warning Centre: Systems. Operations \& Procedures", published by INCOIS, 1-78.

12. Rattaro, C., Larroca, F., Bermolen, P., \& Belzarena, P. (2017). Estimating the medium access probability in large cognitive radio networks. Ad Hoc Networks, 63, 113.

13. P. Vijayalakshmi and V. Rajendran, "Performance Analysis of Fast and Slow Moving Vehicles Using CDMA Techniques for Underwater Wireless Communication Network, Advances in Natural and Applied Sciences, 2016, Special 10(14): pages 92-96.

14. P. Vijayalakshmi, N. Noorunnisa, and V. Rajendran, "Performance Analysis of VBF Protocol for Position Tracking of Moving Nodes in Underwater Communications, Proceeding of International Conference on Communication and Signal Processing, IEEE Xplore, 1813-1817, Feb 2018. doi/10.1109/ICCSP.2017.8286708.

15. R. Rajamohamed, V. Rajamani, 2012, A modified variable bit rate source routing energy efficient protocol for Wireless Mobile adhoc networks, IJEIT, vol.2, Issue $5,177-182$

16. Sehgal, A., Tumar, I., \& Schonwalder, J. (2009, May). Variability of available capacity because of depth and temperature in the underwater acoustic communication channel. In OCEANS 2009-EUROPE (pp. 1-6). IEEE.

17. Sehgal, A., Cernea, D., \& Birk, A. (2010). Simulating underwater acoustic communications in a high-fidelity robotics simulator. IFAC Proceedings Volumes, 43 (16), 587-592.

18. Sozer, E. M., Stojanovic, M., \& Proakis, J. G. (2000), Underwater acoustic networks. IEEE journal of oceanic engineering, 25(1), 72-83

19. The manual published by Kongsberg Simrad, Norway Simrad HiPAP High Precision Acoustic Positioning Product description.

20. Van Heddeghem, W. (2009). Cross layer link estimation for Contiki based wireless sensor networks. Free University Brussels, Faculty Engineering Sciences. 